TITLE:

\title{
Numerical calculation on a top-hat plasma particle analyzer using a boundary-fitted coordinate system
}

\author{
AUTHOR(S):
}

Shimoda, T; Machida, S; Terada, N

\section{CITATION:}

Shimoda, T ... [et al]. Numerical calculation on a top-hat plasma particle analyzer using a boundary-fitted coordinate system. IEEE TRANSACTIONS ON PLASMA SCIENCE 2007, 35(4): 1178-1183

ISSUE DATE:

2007-08

URL:

http://hdl.handle.net/2433/49986

\section{RIGHT:}

(c)2007 IEEE. Personal use of this material is permitted. However, permission to reprint/republish this material for advertising or promotional purposes or for creating new collective works for resale or redistribution to servers or lists, or to reuse any copyrighted component of this work in other works must be obtained from the IEEE. 


\title{
Numerical Calculation on a Top-Hat Plasma Particle Analyzer Using a Boundary-Fitted Coordinate System
}

\author{
Tadahiro Shimoda, Shinobu Machida, and Naoki Terada
}

\begin{abstract}
A boundary-fitted coordinate system is applied to numerical calculation on a top-hat plasma particle analyzer to include the effect of tiny serrations on toroidal plates. The calculation of electrostatic potential inside the analyzer showed the validity of replacement of the serrated toroidal plates to virtual flat toroidal plates, since the potential between the serrated toroidal plates was almost flat except for the area near both the serrated plates. Virtual flat toroidal plates equivalent to serrated plates were obtained from the results of an electric field between the serrated toroidal plates whose position was consistent with an ordinary empirical model. However, calculation of the trajectories of numerous protons revealed sensitivity difference between analyzers with serrated toroidal plates and flat toroidal plates. Such difference was reduced by introducing a path of protons whose boundary is identical to the envelopes of serration tips.
\end{abstract}

Index Terms-Boundary-fitted coordinate system, instrumental development, numerical calculation, plasma measurement.

\section{INTRODUCTION}

A TOP-HAT electrostatic analyzer [1] is one of the most common instruments for obtaining velocity distribution functions of space plasmas. It has been boarded on many satellites such as Freja [2] and Planet-B [3]. To develop it, the shape, size, and other parameters should be optimized depending on the measured plasma environment and the structure of the spacecraft. Numerical simulations have commonly been used for optimization.

When performing numerical simulations, an orthogonal cylindrical coordinate system is usually adopted because it is easy to program, and it quickly calculates the analyzer performance. However, this coordinate system cannot deal with such fine structures as serrations, which are almost always deployed to prevent solar UV from impinging into the deep part of the analyzer. Therefore, they have been replaced by flat plates based on the empirical skill of developers in numerical simulations. In addition, the relationship between such serrations and flat plate has not been numerically investigated yet.

Manuscript received August 18, 2006. This research was supported in part by the Ground-based Research Program for Space Utilization promoted by the Japan Space Forum.

T. Shimoda and S. Machida are with the Department of Geophysics, Graduate School of Science, Kyoto University, Kyoto 606-8502, Japan.

$\mathrm{N}$. Terada is with the National Institute of Information and Communications Technology, Koganei, Tokyo 184-8795, Japan.

Color versions of one or more of the figures in this paper are available online at http://ieeexplore.ieee.org.

Digital Object Identifier 10.1109/TPS.2007.897570

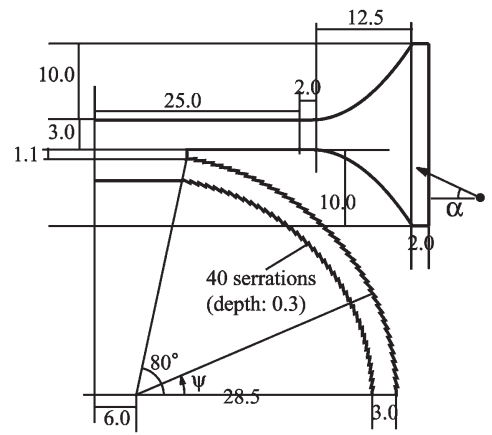

(a)

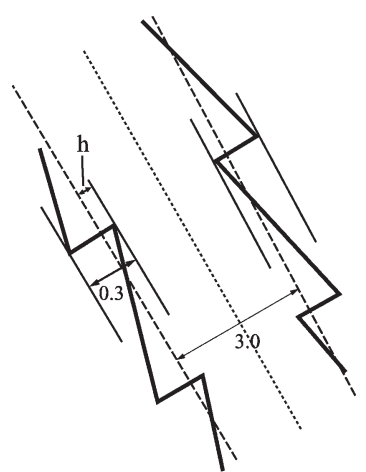

(b)
Fig. 1. Top-hat electrostatic analyzer considered in this paper. Size of analyzer is shown in millimeters. (a) Whole structure of analyzer and a particle entering analyzer with elevation angle of $\alpha$. Due to axial symmetry of the analyzer, only the right part is shown. $\psi$ represents polar angle of toroidal plates. (b) Size and location of serrations on toroidal plates. Serrations are solid lines, and virtual flat plates are dashed lines. Dotted line between both plates is the midpoint line of both plates (midline in text).

To overcome such drawbacks, we adopted a boundary-fitted coordinate system [4], [5], which is a structured grid system whose boundary area is mapped to lines or planes parallel to a coordinate axis. In addition, since the intervals of grid lines can be arbitrarily determined, it can deal with small structures that cannot be considered in an orthogonal coordinate system. We calculated the internal electric field, the detection properties for impinging particles, and the sensitivities of a top-hat electrostatic analyzer with tiny serrations using the boundaryfitted coordinate system and discussed the effect of serrations for deriving an equivalence relationship between the serrated toroidal and flat plates.

The top-hat electrostatic analyzer considered in this paper is shown in Fig. 1. It has an axially symmetric shape, and an electrostatic deflector is placed in front of the top-hat collimator to detect the protons that impinge from various elevation angles, which are represented by $\alpha$ in Fig. 1(a). It carries out the 3-D measurements of proton velocity even if the analyzer is boarded on three-axial stabilized (i.e., nonspin) spacecraft, although the field-of-view coverage is not full 3-D $(<4 \pi)$. The value of $h$ in Fig. 1(b) is variable. At first, we set $h=0.06 \mathrm{~mm}$.

The primary objective of this paper is not analyzer optimization but to develop the method of numerical calculations of electric field, detection properties, and sensitivity. Therefore, analyzer size is not necessarily optimized. 


\section{Calculation Method}

We calculated the analyzer performance in the following ways: generating lattice points on and inside the analyzer, obtaining the internal electric field by solving Laplace's equation for each lattice point, and calculating the trajectories of a number of protons with different parameters that enter the analyzer.

\section{A. Grid Distribution}

The first step is to generate lattice points. This is necessary for a boundary-fitted coordinate system because the grid cells are not generally and equally spaced rectangles, as in a traditional cylindrical coordinate system. First, initial lattice points on the boundary of the analyzer are given, and then, the initial lattice points inside are obtained using a transfinite interpolation method [4], [6]. Next, the initial grid lines are smoothed by solving the Poisson's equations, which are used for electricfield calculation. Since the logical shape is complicated, it was disassembled into parts when generating the initial lattice points and reassembled when smoothing the grid lines. Hereafter, $\boldsymbol{x}=(r, z)$ represents a position in the cylindrical coordinate system, and $\xi$ and $\eta$ are the components of the boundary-fitted coordinate $\left(0 \leq \xi \leq \xi_{\max }, 0 \leq \eta \leq \eta_{\max }\right)$. $\xi$ and $\eta$ are defined as $\Delta \xi=1$ and $\Delta \eta=1$ between the neighboring lattice points in the $\xi(\eta)$ direction. Since the initial lattice points on the boundary are already given, the initial lattice positions inside boundary $\boldsymbol{x}_{0}(\xi, \eta)\left(1 \leq \xi \leq \xi_{\max }-1,1 \leq \eta \leq \eta_{\max }-1\right)$ are calculated in two steps using [6]

$$
\boldsymbol{x}^{(1)}(\xi, \eta)=\alpha(\xi) \boldsymbol{x}_{0}(0, \eta)+\{1-\alpha(\xi)\} \boldsymbol{x}_{0}\left(\xi_{\max }, \eta\right)
$$

and

$$
\begin{aligned}
\boldsymbol{x}_{0}(\xi, \eta)= & \boldsymbol{x}^{(1)}(\xi, \eta)+\beta(\eta)\left\{\boldsymbol{x}_{0}(\xi, 0)-\boldsymbol{x}^{(1)}(\xi, 0)\right\} \\
& +\{1-\beta(\eta)\}\left\{\boldsymbol{x}_{0}\left(\xi, \eta_{\max }\right)-\boldsymbol{x}^{(1)}\left(\xi, \eta_{\max }\right)\right\}
\end{aligned}
$$

where $\alpha(\xi)$ and $\beta(\eta)$ are blending functions, and since we give lattice positions at regular intervals, they are given as follows:

$$
\begin{aligned}
\alpha(0) & =\beta(0)=1 \\
\alpha(\xi) & =1-\frac{s_{1}(\xi)}{s_{1}\left(\xi_{\max }\right)}, \quad \beta(\eta)=1-\frac{s_{2}(\eta)}{s_{2}\left(\eta_{\max }\right)} \\
s_{1}(\xi) & =\sum_{j=1}^{\xi} \sqrt{\left(r_{j, 0}-r_{j-1,0}\right)^{2}+\left(z_{j, 0}-z_{j-1,0}\right)^{2}} \\
s_{2}(\eta) & =\sum_{k=1}^{\eta} \sqrt{\left(r_{0, k}-r_{0, k-1}\right)^{2}+\left(z_{0, k}-z_{0, k-1}\right)^{2}} .
\end{aligned}
$$

To smooth the grid lines, the following Poisson's equations $\partial^{2} \xi / \partial r^{2}+\partial^{2} \xi / \partial z^{2}=P(\xi, \eta)$ and $\partial^{2} \eta / \partial r^{2}+\partial^{2} \eta / \partial z^{2}=$ $Q(\xi, \eta)$ are used, which are solved iteratively. Source terms $P$ and $Q$ are given empirically to prevent grid lines from being strongly distorted.
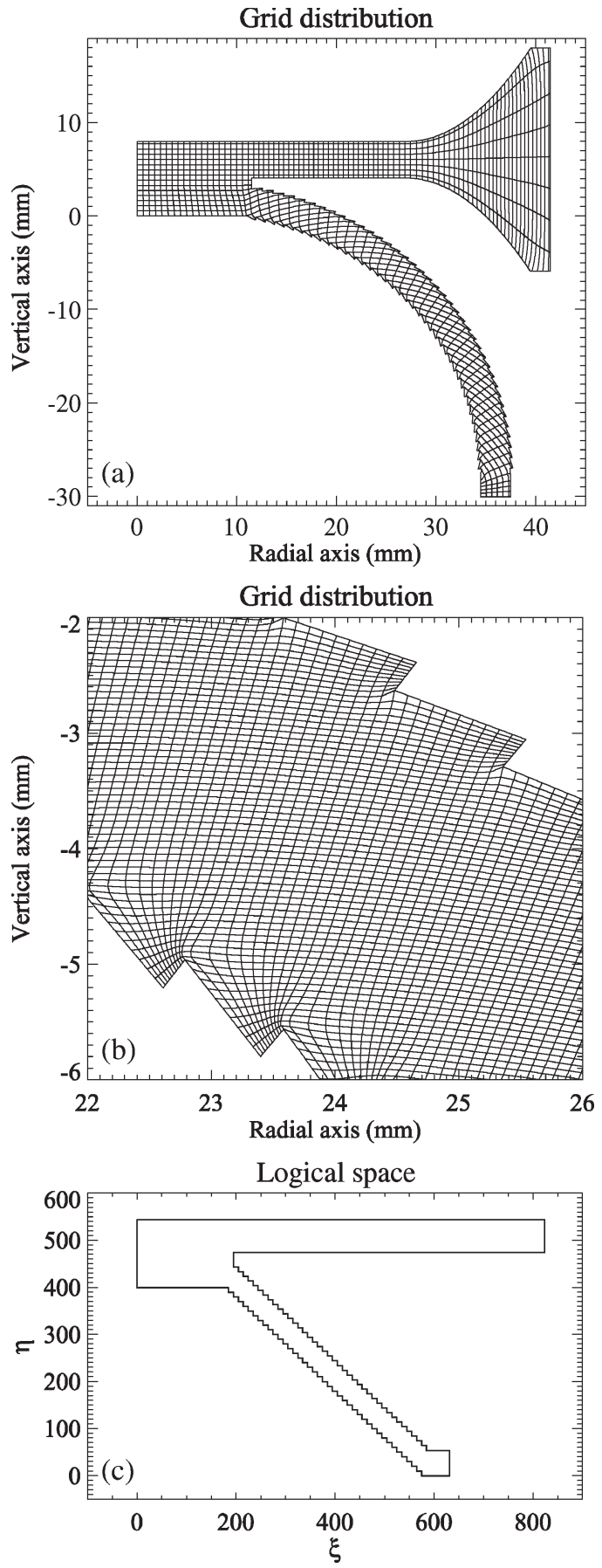

Fig. 2. (a) Grid distribution of particle analyzer in physical space. It is shown in cylindrical coordinate system, and one of the ten grid lines is drawn. (b) Grid lines between the serrated toroidal plates. (c) Shape of analyzer in logical space. $\xi$ and $\eta$ are expressed in grid numbers.

Fig. 2 shows the analyzer's grid distribution and logical shape, i.e., its shape in the boundary-fitted coordinate system. (a) Top panel is the grid distribution of the entire analyzer, (b) middle panel is between the serrated toroidal plates, and (c) bottom panel is the logical shape of the analyzer. One grid line out of ten is drawn in (a). In (c), the left side of the logical space corresponds to the symmetric axis of the analyzer, and small steplike structures correspond to the serrations on the toroidal plates. 


\section{B. Internal Electric Field}

The next step is to calculate the electrostatic field inside the analyzer by solving Laplace's equation. Since we are using a boundary-fitted coordinate system, Laplace's equation expressed with a cylindrical coordinate system should be modified into the following form

$$
\begin{gathered}
\frac{1}{r} J^{-1}\left(z_{\eta} \frac{\partial \Phi}{\partial \xi}-z_{\xi} \frac{\partial \Phi}{\partial \eta}\right) \\
+J^{-1} z_{\eta}\left(\frac{\partial J^{-1}}{\partial \xi} z_{\eta} \frac{\partial \Phi}{\partial \xi}+J^{-1} z_{\xi \eta} \frac{\partial \Phi}{\partial \xi}+J^{-1} z_{\eta} \frac{\partial^{2} \Phi}{\partial \xi^{2}}\right. \\
\left.-\frac{\partial J^{-1}}{\partial \xi} z_{\xi} \frac{\partial \Phi}{\partial \eta}-J^{-1} z_{\xi \xi} \frac{\partial \Phi}{\partial \eta}-J^{-1} z_{\xi} \frac{\partial^{2} \Phi}{\partial \xi \partial \eta}\right) \\
-J^{-1} z_{\xi}\left(\frac{\partial J^{-1}}{\partial \eta} z_{\eta} \frac{\partial \Phi}{\partial \xi}+J^{-1} z_{\eta \eta} \frac{\partial \Phi}{\partial \xi}+J^{-1} z_{\eta} \frac{\partial^{2} \Phi}{\partial \xi \partial \eta}\right. \\
\left.-\frac{\partial J^{-1}}{\partial \eta} z_{\xi} \frac{\partial \Phi}{\partial \eta}-J^{-1} z_{\xi \eta} \frac{\partial \Phi}{\partial \eta}-J^{-1} z_{\xi} \frac{\partial^{2} \Phi}{\partial \eta^{2}}\right) \\
+J^{-1} r_{\eta}\left(\frac{\partial J^{-1}}{\partial \xi} r_{\eta} \frac{\partial \Phi}{\partial \xi}+J^{-1} r_{\xi \eta} \frac{\partial \Phi}{\partial \xi}+J^{-1} r_{\eta} \frac{\partial^{2} \Phi}{\partial \xi^{2}}\right. \\
\left.-\frac{\partial J^{-1}}{\partial \xi} r_{\xi} \frac{\partial \Phi}{\partial \eta}-J^{-1} r_{\xi \xi} \frac{\partial \Phi}{\partial \eta}-J^{-1} r_{\xi} \frac{\partial^{2} \Phi}{\partial \xi \partial \eta}\right) \\
-J^{-1} r_{\xi}\left(\frac{\partial J^{-1}}{\partial \eta} r_{\eta} \frac{\partial \Phi}{\partial \xi}+J^{-1} r_{\eta \eta} \frac{\partial \Phi}{\partial \xi}+J^{-1} r_{\eta} \frac{\partial^{2} \Phi}{\partial \xi \partial \eta}\right. \\
\left.-\frac{\partial J^{-1}}{\partial \eta} r_{\xi} \frac{\partial \Phi}{\partial \eta}-J^{-1} r_{\xi \eta} \frac{\partial \Phi}{\partial \eta}-J^{-1} r_{\xi} \frac{\partial^{2} \Phi}{\partial \eta^{2}}\right)=0
\end{gathered}
$$

where $\Phi$ is the electrostatic potential, and $J^{-1} \equiv$ $\partial r / \partial \xi \partial z / \partial \eta-\partial r / \partial \eta \partial z / \partial \xi$ is the Jacobian inverse of the transformation matrix.

Electrostatic potential is normalized by the voltage applied to the inner toroidal plates. It is fixed to $-1000 \mathrm{~V}$ in this paper, although it varies, in practice, to measure the protons of different energy. The boundary condition used is as follows. The voltage given to the inner toroidal plate is unity due to normalization of voltage. The outer toroidal plate, the tophat collimator, the lower deflector, and the entrance mesh are grounded. The electric potential of the upper deflector is -2 . Potential distribution at the exit of the toroidal plates is identical to the distribution inside the spherical condenser plates. On the insulators, electric potential is given assuming a cylindrical capacitor.

\section{Detection Properties and Sensitivity}

After obtaining the electric field inside the analyzer, the detection properties and sensitivity of the analyzer are calculated by examining whether each of the numerous protons with different parameters travels through the analyzer. The following four parameters describe the initial velocity and position of the incident protons: kinetic energy $(K)$, elevation angle $(\alpha)$ shown in Fig. 1, deviation angle $(\beta)$, which is out of the plane angle of the proton velocity in Fig. 1, and incident height $(z)$.

Particle position is determined by the fourth-order Runge-Kutta method in the cylindrical coordinate system. Then, the electrostatic field must be calculated at the particle position at each time step, and a particle-in-cell (PIC) method coupled by a boundary-fitted coordinate system [5] is adopted for it.

Suppose particle position $\boldsymbol{x}=(r, z)$ corresponds to $(\xi, \eta)=$ $\left(J+\alpha_{1}, K+\alpha_{2}\right)$ in the boundary-fitted coordinate system. Parameters $J$ and $K$ are both integers, and $\alpha_{1}$ and $\alpha_{2}$ should satisfy $0 \leq \alpha_{1}, \alpha_{2}<1$, then $J$ and $K$ are assumed to be the lower left point of the grid cell in which the proton lies and the cell is called cell $(J, K)$ hereafter. Particle position in cell $(J, K)\left(\alpha_{1}\right.$ and $\left.\alpha_{2}\right)$ in the logical space is given by iteratively solving

$$
\begin{aligned}
\alpha_{2}^{0} & =\frac{z^{s}}{1+r^{s} / r_{j+1, k+1}^{s}\left(z_{j+1, k+1}^{s}-1\right)} \\
\alpha_{2}^{i} & =\frac{z^{s}\left\{1+\alpha_{2}^{i-1}\left(r_{j+1, k+1}^{s}-1\right)\right\}}{1+\alpha_{2}^{i-1}\left(r_{j+1, k+1}^{s}-1\right)+r^{s}\left(z_{j+1, k+1}^{s}-1\right)} \\
\alpha_{1} & =\frac{r^{s}}{1+\alpha_{2}\left(r_{j+1, k+1}^{s}-1\right)}
\end{aligned}
$$

where

$$
\left(\begin{array}{c}
r^{s} \\
z^{s}
\end{array}\right) \equiv\left(\begin{array}{ll}
r_{j+1, k}-r_{j, k} & r_{j, k+1}-r_{j, k} \\
z_{j+1, k}-r_{j, k} & z_{j, k+1}-r_{j, k}
\end{array}\right)^{-1}\left(\begin{array}{c}
r-r_{j, k} \\
z-z_{j, k}
\end{array}\right)
$$

and $r_{j, k}, z_{j, k}$ denote $r(j, k), z(j, k)$, respectively. In this paper, iteration was conducted three times, i.e., $\alpha_{2}=\alpha_{2}^{3}$, which is sufficient for calculating the particle position [5]. Then, the electric field at particle position $\boldsymbol{E}$ is obtained by [5]

$$
\begin{aligned}
\boldsymbol{E}=\left(1-\alpha_{1}\right)\left(1-\alpha_{2}\right) \boldsymbol{E}_{j, k}+\alpha_{1}\left(1-\alpha_{2}\right) \boldsymbol{E}_{j+1, k} \\
+\left(1-\alpha_{1}\right) \alpha_{2} \boldsymbol{E}_{j, k+1}+\alpha_{1} \alpha_{2} \boldsymbol{E}_{j+1, k+1}
\end{aligned}
$$

where $\boldsymbol{E}_{j, k}$ means $\boldsymbol{E}\left(r_{j, k}, z_{j, k}\right)$.

After calculating the trajectory of each proton, the detection properties and sensitivity of the analyzer can be obtained. In this paper, energy-alpha characteristics and the $g$-factor are adopted and calculated by using the transmission function $P(K, \alpha, \beta, z)$, where $P(K, \alpha, \beta, z)=1$ if the proton reaches the exit of the analyzer and $P(K, \alpha, \beta, z)=0$ if it does not. Energy-alpha characteristic $C(K, \alpha)$ is a detection property that indicates the acceptance of particles with specific $K$ and $\alpha$. It is obtained by integrating $P(K, \alpha, \beta, z)$ in relation to $\beta$ and $z$, i.e.,

$$
C(K, \alpha)=\sum_{\beta} \sum_{z} P(K, \alpha, \beta, z) .
$$


The $g$-factor $(\mathcal{G})$, which represents the sensitivity of the analyzer, is calculated by

$$
\begin{aligned}
\mathcal{G}= & R \Theta\left\{\int_{0}^{\infty} d K \int_{-\frac{\pi}{2}}^{\frac{\pi}{2}} d \alpha \int_{-\pi}^{\pi} d \beta \int_{-\infty}^{\infty} d z \cos ^{2} \alpha \cos \beta P(K, \alpha, \beta, z)\right\} \\
& /\left\{\int_{0}^{\infty} d K \int_{-\frac{\pi}{2}}^{\frac{\pi}{2}} d \alpha \int_{-\pi}^{\pi} d \beta \int_{-\infty}^{\infty} d z K \cos ^{2} \alpha \cos \beta P(K, \alpha, \beta, z)\right\}
\end{aligned}
$$

where $R$ and $\Theta$ represent the radius at the entrance of particles and the angular width of a sector of microchannel plate (MCP), respectively. In this paper, $\Theta=22.5^{\circ}$.

This study dealt with as many as five million protons that have 64 different energy values varying logarithmically from 4500 to $7500 \mathrm{eV}, 46$ elevation angle values from $11^{\circ}$ to $26^{\circ}$, 18 deviation angle values from $0^{\circ}$ to $17^{\circ}$ (considering axial symmetry of the analyzer), and 100 incident height values from the top to the bottom of the electrostatic deflector in front of the top-hat plates. Elevation angle, deviation angle, and incident position values are distributed at equal intervals in linear scale, except for the incident energy in logarithmic scale. Their range was determined by running the particle calculation in a wider range but with coarse intervals of parameters. In this paper, the analyzer accepts the upward protons $(\alpha>0)$ since the electrostatic deflector makes the particle velocity on the plane of top-hat plates.

\section{Equivalent Plates}

The effect of serrations is considered by deriving a pair of virtual flat toroidal plates equivalent to serrated toroidal plates. Hereafter, virtual flat plates are called equivalent plates.

The positions of the equivalent plates are determined by considering the electric field inside the flat toroidal plates. The intensity of electric field $E$ inside the flat toroidal plates at distance $r_{\mathrm{c}}$ from the curvature center of toroidal plates and polar angle $\psi$, which is zero at the exit of the toridal plates and grows larger as we retreat back deeper from the exit (shown in Fig. 1), is approximately given by:

$$
E\left(r_{\mathrm{c}}\right)=\frac{V}{\ln \frac{R_{\mathrm{o}}\left(R_{\mathrm{i}} \cos \psi+l\right)}{R_{\mathrm{i}}\left(R_{\mathrm{o}} \cos \psi+l\right)}}\left(\frac{1}{r_{\mathrm{c}}}-\frac{1}{r_{\mathrm{c}}+\frac{l}{\cos \psi}}\right)
$$

where $R_{\mathrm{i}}, R_{\mathrm{o}}, l$, and $V$ are the curvature radii of the inner and outer toroidal plates, the offset of curvature center from the symmetrical axis of the analyzer, and the voltage applied to the inner toroidal plate, respectively. We assume that the midpoint line between the equivalent plates corresponds to the midpoint line between both the serrated toroidal plates ("midline" hereafter), which is shown as the dotted line in Fig. 1(b), and obtained the gap width between the equivalent plates $d$ by using the electric field on the midline, i.e., $r_{\mathrm{c}}=$ $30 \mathrm{~mm}$ and various $\psi$ obtained by the calculations of this paper.

We carried out the calculation described previously, and the results are shown in the next section.
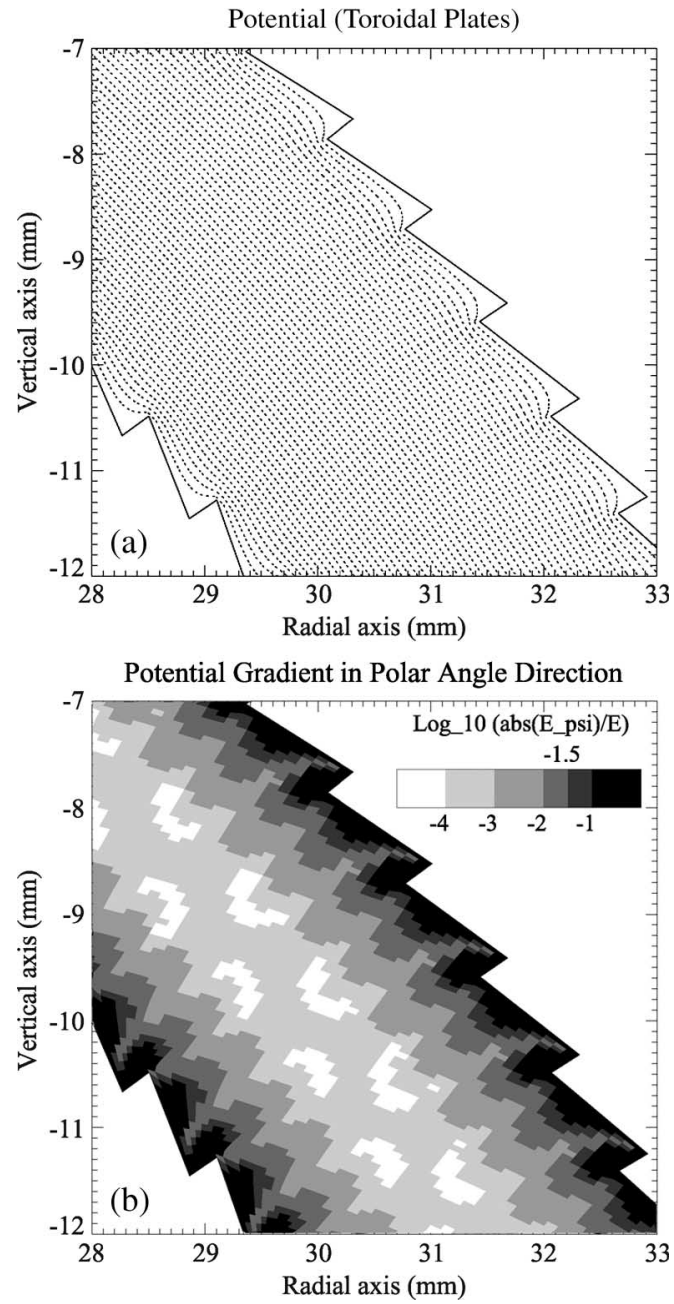

Fig. 3. (a) Electrostatic potential between the serrated toroidal plates. Potential gap between the neighboring contours is 0.02 times the voltage on inner toroidal plate. (b) Component of electric field along the midline normalized by intensity of electric field.

\section{RESULTS}

\section{A. Electrostatic Field and Equivalent Plates}

We solved (6) to obtain the electrostatic potential inside the analyzer in the case of $h=0.06 \mathrm{~mm}$ in Fig. 1. The distribution of electrostatic potential between the serrated toroidal plates is shown in Fig. 3(a). The potential is represented as equipotential contours, and the potential gap between the neighboring contours is 0.02 times the voltage applied to the inner toroidal plates.

Equipotential contours are basically flat in most areas if we go slightly away from the serrated plates. In areas close to the serrations, the contours have ripples. To investigate them quantitatively, we evaluated the value of $\left|E_{\psi}\right| / E$, and the result is shown in Fig. 3(b). The value of $\left|E_{\psi}\right| / E$ represents the tilt of equipotential contours from the midline, where $E_{\psi}$ indicates the electric-field component along the midline (polar angle direction in Fig. 1). The value of $\left|E_{\psi}\right| / E$ is less than $1 \%$ in the region away from the surface of the serrations by about twice the serration depth, and the area at which it is more than $3 \%$ is confined to a region near the length of the serration depth from the surface of the serrations. Therefore, the electric field inside 

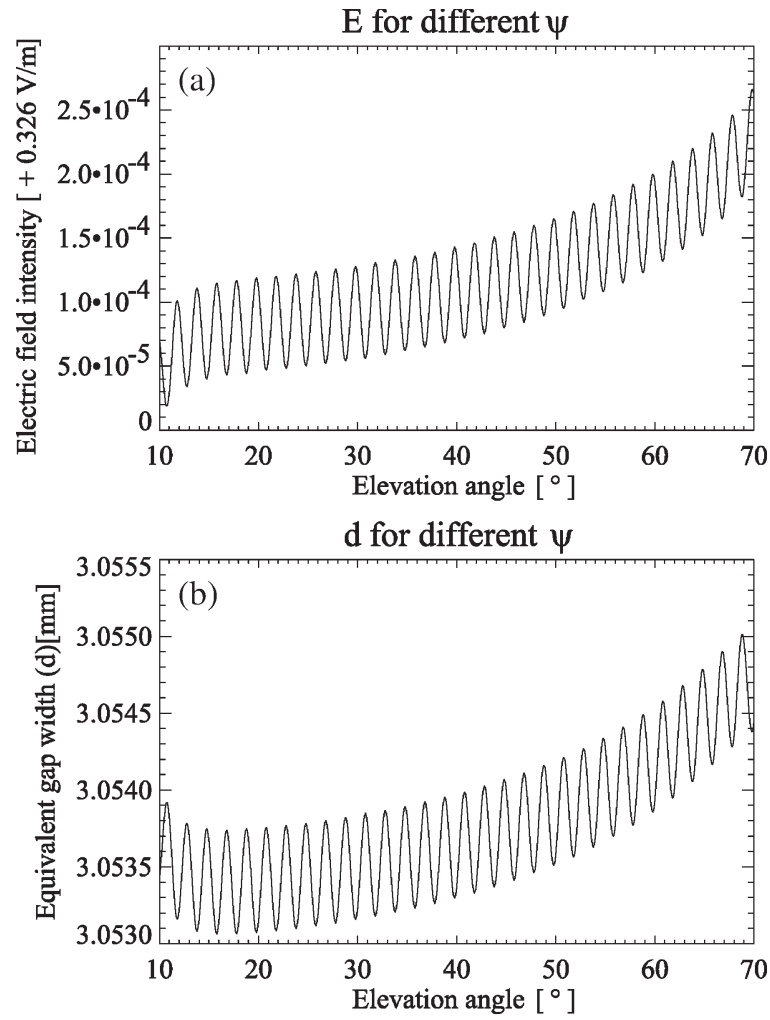

Fig. 4. (a) Electric-field intensity between the serrated toroidal plates for different $\psi$. (b) Gap width between the equivalent plates for different $\psi$, which is calculated by the electric-field intensity shown in (a).

the serrated toroidal plates is approximated by the flat toroidal plates, which ensure that such a replacement is valid.

We now consider the equivalent plates using the obtained electric field between the serrated toroidal plates and (14). We calculated the gap width between the equivalent plates, and the result is shown in Fig. 4. It demonstrates the electric field $E$ in (a) and gap width of equivalent plates $d$ in (b), which was obtained by substituting the electric-field intensity at $r_{\mathrm{c}}=30 \mathrm{~mm}$ and every $0.1^{\circ}$ of $\psi$ inside the serrated toroidal plates. Points near both ends of the toroidal plates $\left(\psi<10^{\circ}\right.$, $\psi>70^{\circ}$ ) were excluded due to the distortion of the electric field there. Fig. 4 shows that the values of $d$ range within $d=3.054 \pm 0.001 \mathrm{~mm}$ for all values of $\psi$, indicating that serrated toroidal plates are replaceable by flat toroidal plates with constant gap width. Comparing the calculated $d$ gap width between the tips of opposite serrations, which is $2.88 \mathrm{~mm}$, and the depth of each serration, which is $0.3 \mathrm{~mm}$, the equivalent plates are placed inward of the serrations from the surface that contains the serration tips by 0.29 times the serration depth. This is consistent with the empirical results where they are placed inward from the surface, including the serration tips roughly 0.2 to 0.3 times the depth of serrations. However, since this replacement only focuses on the electric field, its particle sensitivity has to be verified.

\section{B. Detection Property and Sensitivity of Analyzers}

Energy-alpha characteristics and the $g$-factor of the analyzer with serrated toroidal plates and the scheme with equivalent plates are calculated and compared.
Since $h$ should be 0.29 times the serration depth, it is modified to be 0.087 hereafter. However, we kept the gap width between the equivalent plates to be $3.000 \mathrm{~mm}$, since its alternation affects the position of the neck and the top-hat collimator.

The top and middle rows of Table I show the calculation results for the serrated model described previously and the flat model obtained in the previous section. The $g$-factor, energy, and elevation angle at which the value of $C(K, \alpha)$ is maximum, and the average energy of detected protons is described. The $g$-factors are for $22.5^{\circ}$ of MCP. This result shows that the $g$-factors differ from each other by about $20 \%$, although the energies and elevation angles at the peak of count and the average incident energies of both analyzers are close.

Therefore, we have to modify the equivalent plate to match the $g$-factor with the serrated toroidal plates and obtain correspondence between both analyzers. We restrict the path width of particles between the equivalent plates to effectively modify the $g$-factor without changing the other values.

We examined an analyzer with identical flat toroidal plates, but it contains boundaries for particles so that the gap width between both boundaries is identical to the gap width between the opposite serration tips of the serrated toroidal plates. The result is shown in the bottom row of Table I. Comparing between the top and bottom rows of Table I, the difference in $g$-factor is reduced in several percent. We also show the diagrams of the energy-alpha characteristics in Fig. 5 for analyzers listed in the top and bottom rows of Table I. The energy-alpha characteristics of both analyzers match each other very well. The equivalent plates have almost the same electric field and sensitivity characteristic as the serrated toroidal plates.

\section{DISCUSSION}

By performing the numerical calculation on a top-hat analyzer with serrations, we found virtual flat toroidal plates that were proxyies for the serrated toroidal plates. As a condition for the serrated toroidal plates to be replaced with flat plates, the contours of electrostatic potential around the midline should be almost flat, as shown in Fig. 3(b), which ensures the validity of such substitution.

However, notice that such replacement cannot guarantee the sensitivity of an analyzer that has serrated toroidal plates. This difference can probably be attributed to the gap width between the toroidal plates, which is $2.816 \mathrm{~mm}$ between the opposite serration tips which is narrower than the gap between the flat toroidal plates whose width is $3.000 \mathrm{~mm}$. Protons traveling near the equivalent flat toroidal plates and passing through would hit the serrations if they travel inside the toroidal plates with serrations. This causes the $g$-factors of both analyzers to be different. In addition, when the boundaries for particles are set to the envelopes of the serration tips, the $g$-factors of both analyzers became close to each other.

These facts suggest that the effect of serrations, particularly the ripples of equipotential contours shown in Fig. 3, is insignificant for motions of protons in the analyzers. Ripples of equipotential contours are confined to the area near the serrations, and protons traveling through such area for a long 
TABLE I

Sensitivities and Detection Properties of Analyzers. (Top) Toroidal Plates, Including SerRations. (Middle) With Equivalent Flat Toroidal Plates. (BotTom) Equivalent Flat Toroidal Plates Whose Gap Between the Boundaries

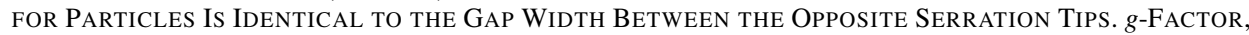
Energy, and Angle at the Peak of Particle Count, and Average Energy Are Shown

\begin{tabular}{ccccc}
\hline \hline Toroidal plates & $\begin{array}{c}\text { g-factor } \\
{\left[\mathrm{cm}^{2} \mathrm{sr} \mathrm{eV} / \mathrm{eV}\right]}\end{array}$ & $\begin{array}{c}\text { (At maximum count) } \\
\text { Energy [eV] }\end{array}$ & $\begin{array}{c}\text { Average } \\
\text { Elev. angle }\left[{ }^{\circ}\right]\end{array}$ & $\begin{array}{c}\text { Energy [eV] } \\
\text { Serrations }\end{array}$ \\
\hline Flat & $9.6818 \times 10^{-4}$ & 5422 & 18.67 & 5537 \\
\hline Flat (limited path) & $7.4560 \times 10^{-4}$ & 5422 & 18.67 & 5533 \\
\hline \hline
\end{tabular}

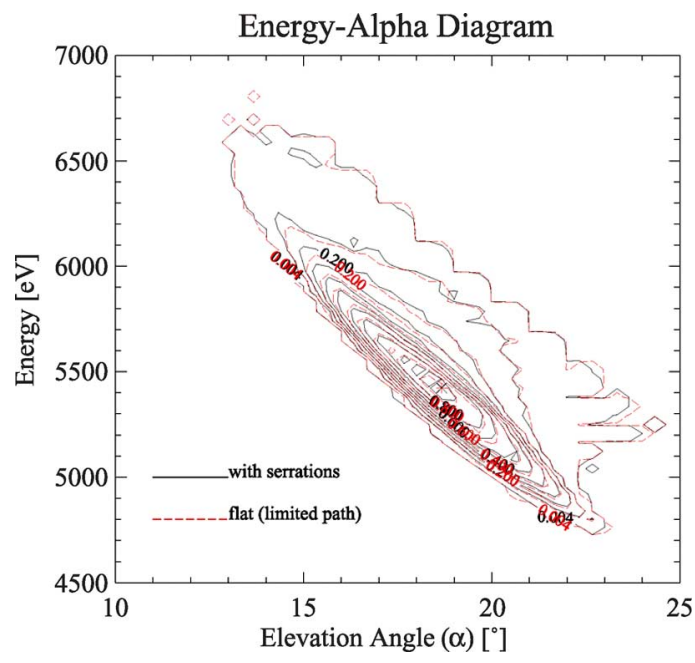

Fig. 5. Energy-alpha diagrams of analyzer with toroidal plates, including (black contour) serrations and (red contour) analyzer with the equivalent flat toroidal plates whose gap between the boundaries for particles is identical to the gap width between the opposite serration tips. Counts are normalized by maximum count of each analyzer.

time are likely to hit a wall of toroidal plates, although they are affected by the rippled electric field. This is supported by the similarity of the energy-alpha characteristics (black and red contours) shown in Fig. 5.

In this paper, we considered the effective figures on the order of micrometer, although it is impossible to make analyzers of such accuracy in practice. However, we focused on the relative position between the serrated toroidal and flat plates and 0.29 times the depth of serrations inward and considered such accuracy for our calculations.

In this way, we demonstrated a more sophisticated equivalence relationship between the serrated and flat toroidal plates, adding the boundary of the particle path. This relationship increases the calculation accuracy and speed of particle analyzers.

In this paper, we describe only the case of 5-6-keV protons. This result is also applicable to other energies and other species of particles. Considering the normalization of dimensions, the result for mass $m$, charge $q$, and particle energy $E$ is applicable to particles with mass $a m$, charge $b q$, and particle energy $c E$ to change the voltage on all plates by $a c / b$ times, where $a, b$, and $c$ are the multiplying factors.

Although we only focused on the tiny serrations on toroidal plates, this coordinate system is applicable to the calculations for other tiny structures, for example, rings on the neck of analyzers and the collimator on the top-hat plane to reduce the effect of secondary electrons by energetic particles. The effect of such structures on the electric field, the detection properties, and the sensitivity of the analyzer must be considered in the future.

\section{REFERENCES}

[1] C. W. Carlson, D. W. Curtis, G. Paschmann, and W. Michael, "An instrument for rapidly measuring plasma distribution functions with high resolution," Adv. Space Res., vol. 2, pp. 67-70, 1983.

[2] L. Eliasson, O. Norberg, R. Lundin, K. Lundin, S. Olsen, H. Borg, M. André, H. Koskinen, P. Rihelä, M. Boehm, and B. Whalen, "The Freja hot plasma experiment-instrument and first results," Space Sci. Rev., vol. 70, pp. 563-576, 1994.

[3] S. Machida, Y. Saito, Y. Ito, and H. Hayakawa, "Instrumental characteristics of the Electron Spectrum Analyzer (ESA) onboard the planet-B mission and observational perspectives of the electron measurements," Earth Planets and Space, vol. 50, pp. 207-211, 1998.

[4] J. F. Thompson, Z. U. A. Warsi, and C. W. Mastin, Numerical Grid Generation Foundations and Applications. New York: Elsevier Science Publishing, 1985.

[5] T. Westermann, "Numerical modelling of the stationary Maxwell-Lorentz system in technical devices," International Journal of Numerical Modelling: Electronic networks, Devices and Fields, vol. 7, pp. 43-67, 1994.

[6] S. Murakami, Grid Generations and Computer Graphics, ser. Computational Fluid Dynamics Series. Tokyo: University of Tokyo Press, 1995. no. 6.

Tadahiro Shimoda was born in Tochigi, Japan, in 1980. He received the M. Sci. degree from Kyoto University, Kyoto, Japan, in 2004. He is currently working toward the Ph.D. degree at Kyoto University. His study involves the measurement of space plasma and spacecraft-plasma interaction in the Earth's magnetosphere.

He was with the Mitsubishi Electric Corporation, Hyogo, Japan, from April 2007.

Shinobu Machida was born in Tokyo, Japan, in 1952. He received the Ph.D. degree in geophysics from the University of Tokyo, Tokyo, Japan, in 1982.

Since 1993, he has been an Associate Professor in the Department of Geophysics, Kyoto University, Kyoto, Japan, and was promoted as Professor in 2000. His research interests include the space plasma physics and space plasma measurements.

Naoki Terada was born in Osaka, Japan, in 1973. He received the D.Sc. degree from Kyoto University, Kyoto, Japan in 2002.

Since 2005, he has been a Core Research for Evolutional Science and Technology (CREST) Research Fellow in the Japan Science and Technology Agency with the Applied Electromagnetic Research Center, National Institute of Information and Communications Technology, Koganei, Japan. His research interests include the interaction between the solar wind and planetary upper atmospheres. 